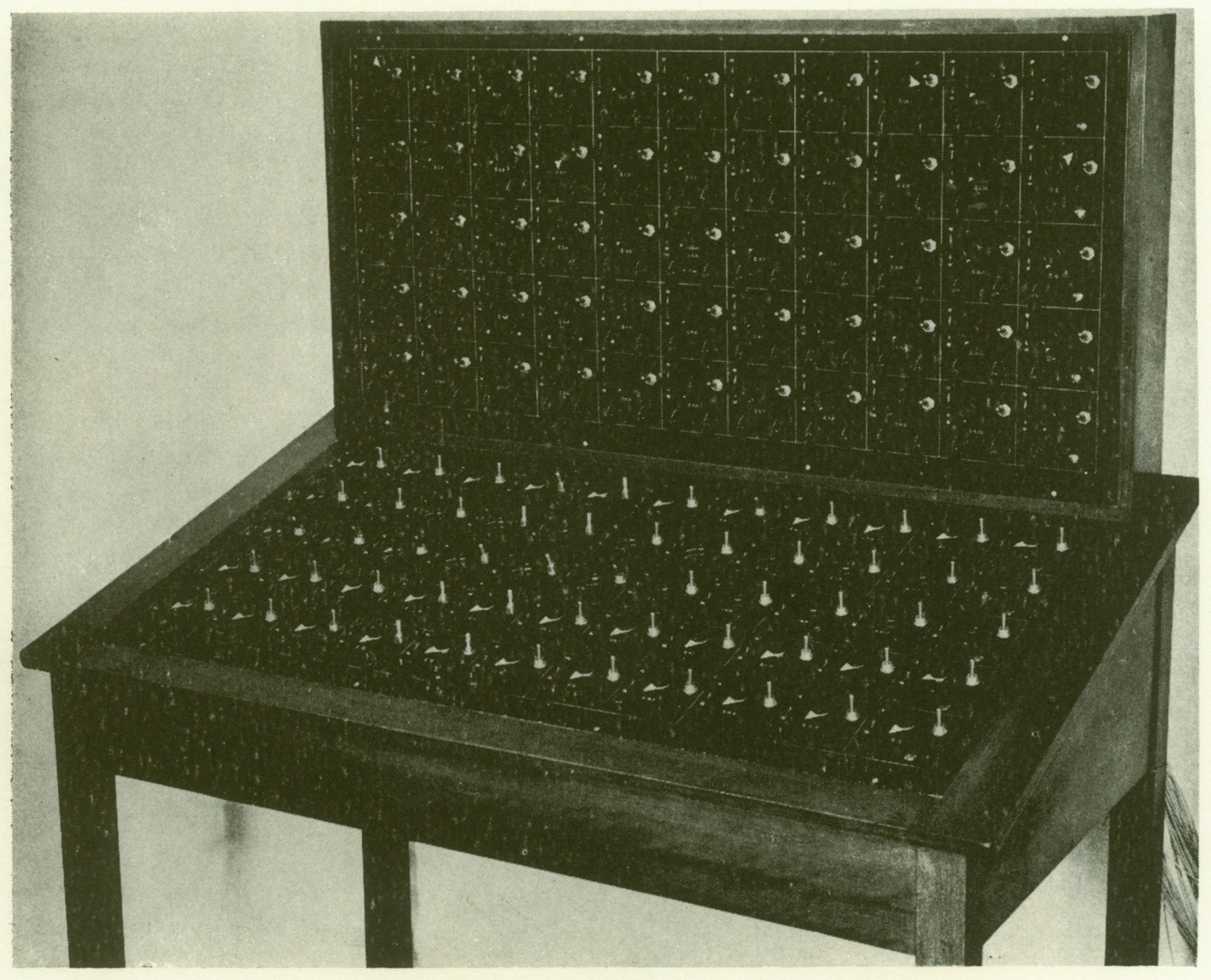

COEFFICIENT MATRIX PANELS 


\section{Use of Continued Fractions in High Speed Computing}

1. Introduction. In the course of carrying out computations required for numerical solution of problems it is frequently necessary to have available the value of one or more functions for various values of their arguments. If a high speed machine is being used, it is usually not efficient to look up tables of the functions outside the machine or store them in the internal memory and it is therefore necessary to calculate the values of the function whenever they are required. This is generally done either by using a rational approximation to the function or a finite number of terms of an infinite process. Usually the infinite process used is that of the power series. The purpose of this paper is to show that another infinite process, a continued fraction expansion, may, in some cases, be more efficient.

The choice of which infinite process to use may depend on :

1. Properties of the method of computing, such as

(a) the number of orders required to program the calculation

(b) rounding error involved

(c) the magnitude of the numbers entering into the calculations

2. Properties of the series, such as

(d) speed of convergence

(e) region of convergence

3. Properties of the machine being used, such as

(f) amount of high speed storage available

(g) what factors determine the total time spent on a problem.

Since methods of computing continued fractions are not as well known as they deserve to be, the following section will be devoted to examples of three methods of computation. In section 3 the speed of convergence of some particular continued fractions will be compared with that of the corresponding power series and in section 4 we shall discuss some situations in which continued fractions might be used.

2. Computing Continued Fractions. A continued fraction expansion of the function $f(x)$ takes the form

$$
f(x)=b_{0}+\frac{a_{1}}{b_{1}}+\frac{a_{2}}{b_{2}}+\cdots,
$$

where the $a$ 's and $b$ 's may be functions of $x$. For convenience the expansion is usually written in the form

$$
f(x)=b_{0}+\frac{a_{1}}{b_{1}}+\frac{a_{2}}{b_{2}}+\frac{a_{3}}{b_{3}}+\cdots .
$$


The following are examples of such expansions

$$
e^{x}=\frac{1}{1}-\frac{x}{1}+\frac{x}{2}-\frac{x}{3}+\frac{x}{2}-\frac{x}{5}+\cdots
$$

(4) $\ln x=\frac{x-1}{1}+\frac{1^{2}(x-1)}{2}+\frac{1^{2}(x-1)}{3}+\frac{2^{2}(x-1)}{4}$

$$
+\frac{2^{2}(x-1)}{5}+\frac{3^{2}(x-1)}{6}+\cdots
$$

$$
\arctan x=\frac{x}{1}+\frac{(x)^{2}}{3}+\frac{(2 x)^{2}}{5}+\frac{(3 x)^{2}}{7}+\cdots .
$$

In practice a finite number of terms are used to approximate the function; the so-called " $n$th approximant" is given by

$$
f_{n}(x)=b_{0}+\frac{a_{1}}{b_{1}}+\frac{a_{2}}{b_{2}}+\cdots+\frac{a_{n}}{b_{n}} .
$$

Three different ways of computing $f_{n}(x)$ are given by the following methods I, II and III.

Method I. The obvious way to compute $f_{n}(x)$, if $n$ is given, is to carry out the successive additions and divisions indicated by the form of the

TABLE 1. Computation of arc tan 1 using ten terms of the continued fraction

$\begin{array}{rrccc}i & b_{n-i} & d_{n-i} & a_{n-i} & c_{n-i} \\ 0 & 19 & 19 . & 81 & 4.26315789 \\ 1 & 17 & 21.26315789 & 64 & 3.00990099 \\ 2 & 15 & 18.00990099 & 49 & 2.72072567 \\ 3 & 13 & 15.72072567 & 36 & 2.28997063 \\ 4 & 11 & 13.28997063 & 25 & 1.88111778 \\ 5 & 9 & 10.88111778 & 16 & 1.47043717 \\ 6 & 7 & 8.47043717 & 9 & 1.06251895 \\ 7 & 5 & 6.06251895 & 4 & .65979175 \\ 8 & 3 & 3.65979175 & 1 & .27323959 \\ 9 & 1 & 1.27323959 & 1 & .78539814\end{array}$

expansion when it is written as in (1). More formally, the method consists of calculating the sequence

$$
\begin{aligned}
d_{n-i} & =b_{n-i}+c_{n-i+1} \quad c_{n+1}=0 \\
c_{n-i} & =a_{n-i} / d_{n-i}
\end{aligned}
$$

for $i=0,1, \cdots n-1$. Then $f_{n}(x)=b_{0}+c_{1}$. This method is illustrated in Table 1 by the computation of arc tan 1 using 10 terms of expansion (5).

Method II. Successive approximants, for consecutive $n$, may be calculated by writing

where

$$
f_{n}(x)=A_{n} / B_{n},
$$

$$
\begin{array}{lll}
A_{0}=b_{0} & A_{1}=b_{0} b_{1}+a_{1} & A_{n+1}=b_{n+1} A_{n}+a_{n+1} A_{n-1} \\
B_{0}=1 & B_{1}=b_{1} & B_{n+1}=b_{n+1} B_{n}+a_{n+1} B_{n-1} .
\end{array}
$$

An example using this method is given in Table 2 where expansion (4) is used to calculate $\ln 2.3026$. 
TABLE 2. Computation of $\ln 2.3026$ by Method II

\begin{tabular}{|c|c|c|c|c|c|}
\hline$n$ & $b_{n}$ & $a_{n}$ & $A_{n}$ & $B_{n}$ & $A_{n} / B_{n}$ \\
\hline $\mathbf{0}$ & 0 & - & $\mathbf{0}$ & $\times 10^{1}$ & - \\
\hline 1 & 1 & 1.3026 & .13026 & $\times 10^{1}$ & 1.3026 \\
\hline 2 & 2 & 1.3026 & .26052 & .33026 & .788833041 \\
\hline 3 & 3 & 1.3026 & $.951236676 \times 10^{1}$ & .112104 & .848530539 \\
\hline 4 & 4 & 5.2104 & $.5162360112 \times 10^{2}$ & $.62049467 \times 10^{2}$ & .831974933 \\
\hline 5 & 5 & 5.2104 & $.3076812414 \times 10^{3}$ & $.3686580032 \times 10^{3}$ & .834598025 \\
\hline 6 & 6 & 11.7234 & $.2451291574 \times 10^{4}$ & $.2939378741 \times 10^{4}$ & .833948868 \\
\hline 7 & 7 & 11.7234 & $.2076611128 \times 10^{5}$ & $.2489757642 \times 10^{5}$ & .834061554 \\
\hline 8 & 8 & 20.8416 & $.2172177286 \times 10^{6}$ & $.2604419673 \times 10^{6}$ & .834035048 \\
\hline 9 & 9 & 20.8416 & $.2387758543 \times 10^{7}$ & $.2862883034 \times 10^{7}$ & .834039852 \\
\hline 10 & 10 & 32.5650 & $.3095128077 \times 10^{8}$ & $.3711012300 \times 10^{8}$ & .834038754 \\
\hline 11 & 11 & 32.5650 & $.4182214453 \times 10^{9}$ & $.5014411389 \times 10^{9}$ & .834038959 \\
\hline 12 & 12 & 46.8936 & $.5 \times 10^{10}$ & $.7757520931 \times 10^{10}$ & .834038913 \\
\hline 13 & 13 & 46.8936 & $54 \times 10^{12}$ & .1243621 & .834038922 \\
\hline 14 & 14 & 63.8274 & $276 \times 10^{13}$ & $.2236212522 \times 10^{13}$ & .834038919 \\
\hline & 15 & 63.8274 & .3459668 & $.4148090065 \times 10^{14}$ & .834038920 \\
\hline
\end{tabular}

Method III. The $n$th approximant may also be expressed as a sum. i.e.,

$$
f_{n}(x)=b_{0}+\sum_{i=1}^{n} \rho_{1} \rho_{2} \cdots \rho_{i}
$$

where

with initial values

$$
r_{i}=\frac{a_{i}}{b_{i-1} b_{i}} \quad 1+\rho_{i}=\frac{1}{1+r_{i}\left(1-\rho_{i-1}\right)}
$$

$$
\rho_{1}=\frac{a_{1}}{b_{1}} \quad 1+\rho_{2}=\frac{1}{1+r_{2}} .
$$

\begin{tabular}{|c|c|c|c|c|}
\hline$n$ & $r_{n}$ & $1+\rho_{n}$ & $\rho_{1} \rho_{2} \cdots \rho_{n}$ & $f_{n}$ \\
\hline 1 & 1.3026 & 1.0 & 1.3026 & 1.3026 \\
\hline 2 & .6513 & .605583480 & -.513766959 & .7888330 \\
\hline 3 & .2171 & .883804325 & .059697499 & .848530540 \\
\hline 4 & .4342 & .722675023 & -.016555608 & .831974932 \\
\hline 5 & .26052 & .841558660 & .002623093 & .834598025 \\
\hline 6 & .39078 & .752522289 & -.000649157 & .8339488 \\
\hline & .279128571 & .826411810 & .000112686 & .834061554 \\
\hline 8 & .372171429 & .764779246 & -.000026506 & .834035048 \\
\hline 9 & .289466667 & .818747283 & .000004804 & .834039852 \\
\hline 10 & .361833333 & .771456089 & -.000001098 & .834038754 \\
\hline 11 & .296045455 & .814076311 & .000000204 & .8340389 \\
\hline 12 & .355254545 & .775672245 & -.000000046 & .8340389 \\
\hline 13 & .300600000 & .810920125 & .000000009 & .834038921 \\
\hline 14 & .350700000 & .778579904 & -.000000002 & .834038919 \\
\hline 15 & .303940000 & .808641743 & .000000000 & .834038919 \\
\hline
\end{tabular}

Table 3 gives the computation of $\ln 2.3026$ by this method.

TABLE 3. Computation of $\ln 2.3026$ by Method III

3. Truncation Error. These errors arise because only a finite number of terms of an infinite process can be used. For a discussion of these errors it is convenient to separate functions into classes on the basis of the order of magnitude of the $n$th term in their power series expansions. If the order of magnitude of the $n$th term is $x^{n} / n$ ! the function belongs to one class; if it is $x^{n} / n$ the function belongs to a second class, etc. The reason for this classification is that functions in the first class can generally be computed adequately from their power series, while for functions of the second 
class an unreasonable number of terms may be required. HeIsing ${ }^{10}$ states that in computing $\operatorname{arc} \tan x$ by means of a general purpose board on the 604 Electronic Calculating Punch the maximum error after 990 terms are used is still $11 \times 10^{-7}$. Fortunately the continued fractions of some of the functions in the second class converge rapidly enough to make them practical for machine computation.

TABLE 4. Truncation error in $e^{x}$ after 10 terms

$\begin{array}{ccc}x & \text { Power Series } & \text { Continued Fraction } \\ 1 & 2.7 \times 10^{-8} & .67 \times 10^{-8} \\ 2 & 61 \times 10^{-6} & 22 \times 10^{-6} \\ 3 & 59 \times 10^{-4} & 44 \times 10^{-4}\end{array}$

Examples of functions belonging to the first class are $e^{x}, \sin x, \cos x$, $\sinh x$ and $\cosh x$ and examples of functions belonging to the second class are $\ln x, \operatorname{arc} \sin x$, arc $\sinh x, \operatorname{arc} \tan x$ and $\operatorname{arctanh} x$. To compare the convergence of the power series and the continued fractions expansions of functions in these two classes we have chosen $e^{x}$ to represent the first class and $\ln x$ and $\operatorname{arc} \tan x$ to represent the second. The continued fractions expansions are given by (3), (4) and (5) and the power series are

$$
\begin{gathered}
e^{x}=1+\frac{x}{1}+\frac{x^{2}}{2 !}+\frac{x^{3}}{3 !}+\cdots \\
\ln x=\frac{(x-1)}{1}-\frac{(x-1)^{2}}{2}+\frac{(x-1)^{3}}{3}-\cdots \\
\arctan x=\frac{x}{1}-\frac{x^{3}}{3}+\frac{x^{5}}{5}-\cdots
\end{gathered}
$$

Tables 4, 5 and 6 give the comparisons. The calculations were carried out to two more decimal digits than were required, this should make the rounding error negligible (except, perhaps, for the computation of $\ln x$ for $x$ near zero).

TABLE 5. Number of terms required to compute $\ln x$ to 9 decimals

$\begin{array}{ccc}x & \text { Power Series } & \text { Continued Fraction } \\ .0001 & 95,000^{*} & 550 \\ .0010 & 11,500^{*} & 315 \\ .0101 & 1,350^{*} & 105 \\ .1054 & 143 & 34 \\ .5108 & 25 & 16 \\ .6931 & 16 & 11 \\ .9163 & 8 & 7 \\ 2.3026 & - & 16 \\ 4.6052 & - & 24 \\ 6.9078 & - & 30\end{array}$

* Approximate values.

Table 4 gives the difference between $e^{x}$ and the 10th approximant from the power series and from the continued fraction (in the power series the last term is $x^{10} / 10$ !). This table indicates that the continued fraction converges a little faster than the power series but the difference is too small to be of practical importance. 
Table 5 gives a comparison of the number of terms required for fixed accuracy. For the power series the number of terms, $n$, is determined by the condition that the absolute value of the $(n+1)$ th term in $(8)$ shall be less than $9.3132 \times 10^{-10}$. The continued fractions were computed by Method III and $n$ was obtained as the first integer for which $\rho_{1} \rho_{2} \cdots \rho_{n}<9.3132 \times 10^{-10}$. The table shows that the continued fraction expansion converges appreciably faster than the power series in $0<x<2$. Actually the continued fraction converges for all $x>0$, while the power series diverges for $x>2$. The continued fraction could be used to compute, in a reasonable number of terms, $\ln x$ for $.1<x<10$.

Table 6 gives the corresponding comparison for the arc tangent. For the power series, $n$ is determined by the condition that the absolute value of the $(n+1)$ th term in (9) shall be less than $10^{-6}$ while for the continued fraction $n$ is the number of terms required to reduce the truncation error to less than $10^{-6}$. The continued fraction converges faster than the power series and also converges in the region where the power series does not, i.e., for $x$ greater than 1 .

TABLE 6. Number of terms required to compute arc $\tan x$ to 6 decimals

$\begin{array}{ccc}x & \text { Power Series } & \text { Continued Fraction } \\ .1 & 3 & 3 \\ .2 & 4 & 3 \\ .3 & 5 & 4 \\ .4 & 6 & 5 \\ .5 & 8 & 5 \\ .6 & 11 & 7 \\ .7 & 15 & 7 \\ .8 & 22 & 7 \\ .9 & 44 & 8 \\ 1.0 & - & 8 \\ 2.0 & - & 15\end{array}$

4. Machine Computation. Computing machines differ greatly in many aspects. In deciding what method should be used to compute values of functions the amount of high speed storage available is one of the most important factors.

Machines which have enough high speed storage to accommodate arbitrary coefficients can probably compute values of many functions most rapidly by using polynomial approximations with a fixed number of terms. If, however, the majority of the arguments fall in the region in which only a few terms of an infinite process are required, or if varying degrees of accuracy are required from one computation to another, the infinite process may be more efficient. In general the computation of a term in a power series requires fewer operations than the computation of a term in the continued fraction. However for functions such as $\ln x$ and $\operatorname{arc} \tan x$ more terms of the power series are required. In either case it is desirable to compute only as many terms as are required to achieve the required accuracy. Therefore, for computing continued fractions, Method I would not be suitable, but Method II or III could be used. It is immediately evident from Table 2 that Method II cannot, in general, be used without a floating decimal point, because $A_{n}$ and $B_{n}$ increase too rapidly. Furthermore, since 
Method II requires as many operations and more storage than Method III the latter is more' suitable for machine calculations.

Table 7 gives the calculations required in computing successive approximants to the continued fraction, the values required from storage and the initial values, if Method III is used. If $a_{n}$ and $b_{n}$ are simple functions of $n$ and $x$ which can be computed as required, the first calculation requires the storage of only $n$ and $x$. For example, for expansion (4),

$$
r_{n}=\left[\frac{1}{2} n\right]^{2}(x-1) /\{n(n-1)\},
$$

where $[y]$ denotes the greatest integer $\leq y$. Even with this simplification, five quantities must be stored.

This fact makes this method of computation impossible for machines with very small internal memories, such as the IBM 604 Electronic Calculating Punch. The computation of power series requires very little storage and can be carried out on such machines. However for functions of the second class where too many terms of the power series are required, continued fractions can be used if the maximum number of terms of the continued fraction expansion that will be needed to give the desired accuracy is determined in advance and Method $I$ is used to compute the value of the expansion. If the coefficients can be generated in a simple manner only three numbers have to be stored at any one time. ${ }^{11}$

TABLE 7

\begin{tabular}{|c|c|c|}
\hline Calculation & Required from Storage & Initial Values \\
\hline $\begin{array}{l}\text { 1. } \quad r_{n}=a_{n} / b_{n-1} b_{n} \\
\text { 2. } 1+\rho_{n} \stackrel{=}{=}\left\{1+r_{n}\left(1+\rho_{n-1}\right)\right\}^{-1} \\
\left.\text { 3. } \quad \rho_{1} \rho_{2} \cdots \rho_{n-1}\right) \rho_{n} \\
\text { 4. } \quad f_{n}=f_{n-1}+\rho_{1} \rho_{2} \cdots \rho_{n}\end{array}$ & $\begin{array}{c}a_{n}, b_{n-1}, b_{n} \\
1+\rho_{n-1} \\
\rho_{1} \rho_{2} \ldots \rho_{n-1} \\
f_{n-1}\end{array}$ & $\begin{array}{c}1 \\
1 \\
a_{1} / b_{1} \\
b_{0}+a_{1} / b_{1}\end{array}$ \\
\hline
\end{tabular}

5. Bibliography. The general theory of continued fractions is given by PERroN $^{1}$ and Wall. ${ }^{2}$ Both of these books have extensive bibliographies. The use of continued fractions in interpolation is discussed in MILNEThompson, ${ }^{3}$ NÖRLUND $^{4}$ and LANE. ${ }^{5}$ MÜIR $^{6}$ gives some general methods for transforming infinite series into continued fractions. MüLlER, ${ }^{7}$ ArOIAN $^{8}$ and BURGESS $^{9}$ give some examples of the use of continued fractions in evaluating integrals.

University of North Carolina

D. TEICHROEW

Raleigh, N. C.

NBSINA

This work was performed on a National Bureau of Standards contract with the University of North Carolina.

1 O. Perron, Die Lehre von den Kettenbrüchen. New York, 1950.

2 H. S. Wall, The Analytic Theory of Continued Fractions. New York, 1948.

${ }^{3}$ L. M. Milne-Thomson, The Calculus of Finite Differences. London, 1933.

4 N. E. NöRLUND, Vorlesungen über Differenzenrechung. Berlin, 1924, p. 438-55.

- R. E. LANE, "Interpolation by means of continued fractions," Fraternal Actuarial Assoc., Proc., no. 19, 1944-46.

-T. Muir, "New general formulae for the transformation of infinite series into continued fractions," Roy. Soc. Edin., Trans., v. 27, 1872-76, p. 467. 
$7 \mathrm{~J}$. H. MülLLER, “On the application of continued fractions to the evaluation of certain integrals, with special reference to the incomplete Beta function," Biometrika, v. 22, 1920-1, p. 284-297.

${ }^{8}$ L. A. Aroian, "Continued fractions for the incomplete Beta function," Annals Math. Stat., v. 12, 1941, p. 218-23.

'J. Burgess, "On the definite integral $\frac{1}{\pi} \int_{0}^{t} e^{-t^{2}} d t$ with extended tables of values," Roy. Soc. of Edin., Trans., v. 39, part II, 1898, p. 257-321.

io W. P. HeIsING, "An eight-digit general purpose control panel," IBM Technical Newsletter, no. 3, 1951.

1 The use of continued fractions in computing $\ln x, \arctan x$ and $\operatorname{arc} \sin x$ on the Model II Card Programmed Calculator will be discussed in a forthcoming National Bureau of Standards report.

\section{On a Punched-Card Method of Solving Certain Integral Equations}

1. Introduction. In the present paper we are concerned with the numerical solution of the homogeneous Fredholm equation

$$
\phi(y)=\int_{0}^{1} p(x, y) \phi(x) d x,
$$

where the given kernel $p(x, y)$ satisfies the conditions

$$
p(x, y)>0, \quad 0 \leq x \leq 1, \quad 0 \leq y \leq 1
$$

and

$$
\int_{0}^{1} p(x, y) d y=1, \quad 0 \leq x \leq 1 .
$$

The solution $\phi(y)$ is to be a non-negative function such that

$$
\int_{0}^{1} \phi(y) d y=1 .
$$

Equations of the type (1.1) have received considerable attention in the theory of probability, ${ }^{1,2}$ and the above problem has been formulated with this in mind. Although all the examples which have been considered by the authors are special cases of (1.1), the numerical method to be explained is immediately applicable to the following more general equation

$$
\phi(y)=f(y)+\int_{a}^{b} K(x, y, \lambda) \phi(x) d x,
$$

where $f(y), K(x, y, \lambda)$ are given functions, $\lambda$ is a parameter (supposed known) and $a, b$ are constants. This integral equation includes the general non-homogeneous Fredholm equation

$$
\phi(y)=f(y)+\lambda \int_{a}^{b} K(x, y) \phi(x) d x
$$

as a special case.

Numerical methods for the solution of integral equations have been considered by a number of writers..$^{3-10}$ The methods employed may be classified briefly as follows: (a) Methods which involve the solution of a 